Article

\title{
Impact of Weak Nanoparticle Induced Disorder on Nematic Ordering
}

\author{
Dejvid Črešnar ${ }^{1}$, Christina Kyrou ${ }^{2}$, Ioannis Lelidis ${ }^{2}$, Aleksandra Drozd-Rzoska ${ }^{3}$, \\ Szymon Starzonek ${ }^{3}$, , Sylwester Janusz Rzoska ${ }^{3}$, Zdravko Kutnjak ${ }^{1}$ (i) and Samo Kralj ${ }^{1,4, *(1)}$ \\ 1 Jožef Stefan Institute, Jamova 39, 1000 Ljubljana, Slovenia; Dejvid.Cresnar@ijs.si (D.Č.); \\ zdravko.kutnjak@ijs.si (Z.K.) \\ 2 Faculty of Physics, National and Kapodistrian University of Athens, Panepistimiopolis, Zografos, \\ 15784 Athens, Greece; ckyrou@phys.uoa.gr (C.K.); ilelidis@phys.uoa.gr (I.L.) \\ 3 Institute of High Pressure Physics, Polish Academy of Sciences, ul. Sokolowska 29/37, 01-142 Warsaw, \\ Poland; sylwester.rzoska@unipress.waw.pl (A.D.-R.); starzoneks@unipress.waw.pl (S.S.); \\ sylwester.rzoska@gmail.com (S.J.R.) \\ 4 Faculty of Natural Sciences and Mathematics, University of Maribor, Koroška 160, 2000 Maribor, Slovenia \\ * Correspondence: samo.kralj@um.si
}

Received: 28 February 2019; Accepted: 22 March 2019; Published: 24 March 2019

\begin{abstract}
Dilute mixtures of nanoparticles (NPs) and nematic liquid crystals (LCs) are considered. We focus on cases where NPs enforce a relatively weak disorder to the LC host. We use a Lebwohl-Lasher semi-microscopic-type modeling where we assume that NPs effectively act as a spatially-dependent external field on nematic spins. The orientational distribution of locally favoured "easy" orientations is described by a probabilistic distribution function $P$. By means of a mean field-type approach, we derive a self-consistent equation for the average degree of nematic uniaxial order parameter $S$ as a function of the concentration $p$ of NPs, NP-LC coupling strength and $P$. Using a simple step-like probability distribution shape, we obtain the $S(p)$ dependence displaying a crossover behaviour between two different regimes which is in line with recent experimental observations. We also discuss a possible origin of commonly observed non-monotonous variations of the nematic-isotropic phase temperature coexistence width on varying $p$.
\end{abstract}

Keywords: phase behaviour; weak disorder; orientational order

\section{Introduction}

In recent years, there has been an increasing interest in homogeneous composites [1] consisting of soft materials doped with appropriate nanoparticles (NPs). The main goal is to combine constituents possessing complementary properties to obtain composite materials with anomalously enhanced or even new material properties. Diverse mixtures often exhibit complex behaviour, leading to emergent robust, and in some cases even universal behaviour. In this contribution, we focus on the impact of NP-induced weak disorder on anomalous host structural behaviour, in which we exploit nematic liquid crystals as a demonstrative test bed.

Liquid crystals (LC) [2] are typical representatives of anisotropic soft materials. These extraordinary materials combine liquid and crystalline ordering, optic transparency and anisotropy, and softness. The latter refers to the capacity of strong responses to relatively weak perturbations. The reasons behind the softness are weak intermolecular interactions between LC molecules and the formation of LC phases and structures via continuous symmetry breaking (CSB) transitions [3]. Due to the latter, bulk LC phases exhibit easily excitable Goldstone modes in the gauge field component of the relevant order parameter field $[2,3]$. Due to these unique combinations of properties, LC phases and structures are inevitable 
in numerous "natural" applications (e.g. biological membranes [4]), and technological applications (e.g., LC displays [3]).

Several studies of NP-LC mixtures focused on the impact of NP-induced disorder on LC phase and structural behaviour [5]. Namely, due to the experimental accessibility of LCs and their susceptibility to perturbations (in this case NPs), one could exploit the rich diversity of different LC-NP combinations to (i) control and tune disorder type and even disorder strength [6], (ii) and relatively easily measure their impact on macroscopic order [7]. Consequently, LCs represent an ideal testing ground to study the influence of various types of disorder on material properties [8].

Most studies so far have considered the impact of relatively weak or strong random field-type disorders imposed by aerosil NPs on nematic $[6,9,10]$ and smectic A phases $[6,11]$. These phases are the simplest representatives of systems exhibiting orientational and translational degrees of freedom, respectively. These studies focused mainly on the validity of the Imry-Ma argument [12], one of the pivotal cornerstones of the statistical mechanics of disordered systems. It claims that even an infinitesimally weak random field-type disorder destroys the long-range order of the undistorted phase, which is reached via a CSB phase transition. The resulting structures exhibit short-range order. Therefore, the argument applies to any configuration reached via a continuous symmetry breaking the transition in the presence of a random field disorder. However, numerous studies, among which several were performed in NP-perturbed LCs, that in some cases instead of short-range order [12-14], quasi-long range order [13-16] or even long-range order [10,13,14] might appear.

In this paper, we consider cases where NPs induce a weak enough disorder, so that resulting LC structures exhibit qualitatively bulk-like behaviour, but quantitatively complex behaviour on increasing the disorder strength. We consider the phase and structural behaviour of thermotropic nematic LCs doped with NPs of volume concentration $p$. We first discuss recent experimental observations $[17,18]$, which reveal nonmonotonous I-N temperature phase behaviour on varying $p$ in the diluted regime (typically, $p<0.01)$. This was observed $[17,18]$ in $\Delta T(p)=T^{* *}(p)-T^{*}(p) \sim$ $T_{I N}(p)-T^{*}(p)$ dependence, where $T_{I N}(p), T^{* *}(p)$, and $T^{*}(p)$ stands for the I-N phase transition, nematic superheating, and isotropic supercooling temperature, respectively, in mixtures. In these cases, $\Delta T(p)$ first decreases and afterward increases on increasing $p$. Next, we develop a simple model to explain recent experimental results which reveal nontrivial behaviour in bulk uniaxial nematic order parameter $\mathrm{S}$ on varying $p$ in LC doped with spherical NPs [19]. In the paper, we address these phenomena.

\section{Results}

We study thermotropic nematic LCs doped with NPs of volume concentration $p$ in the diluted regime (i.e., $p \ll 1$ ). We consider the two experimental observations addressed above, for which generic mechanism are not known: (i) non-monotonous $\Delta T(p)$ dependence $[17,18]$, and (ii) $p$-driven crossover in $S(p)$ dependence [19]. We first describe a possible mechanism yielding the non-monotonous $\Delta T(p)$ behavior. Afterward we develop a simple phenomenological model originating from the Lebwohl-Lasher-type lattice approach [20-22] to explain crossover behaviour in nematic ordering on varying $p$.

\subsection{Non-Monotonous $\Delta T(p)$ Dependence}

To identify a possible origin of non-monotonous $\Delta T(p)$ behavior, we first discuss the most probable behavior on decreasing temperature across the I-N phase transition for different concentrations of NPs. For this purpose, we consider simple model systems, which roughly mimic the experimentally studied systems in Reference [17-19]. We assume that NPs are essentially spatially homogeneously distributed in the LC medium. Furthermore, the NP-LC coupling is sufficiently weak, so that NPs do not enforce topological defects in the LC medium. We set the NPs to be spherical and enforce a relatively weak homeotropic anchoring [2]. In this case, LC molecules tend to be aligned along the surface normal of an infinitesimally small NP-LC interface area. Due to the weak anchoring 
condition, a local LC ordering reflects the compromise between local elastic forces, surface anchoring, and wetting tendencies.

In our treatment, we use the volume concentration, which is defined as

$$
p=\frac{N v}{V}
$$

where $N, v=4 \pi r^{3} / 3$; and $V$ stands for the number of NPs, the volume of an average spherical NP, and sample volume, respectively. In addition to the NP radius $r$, there are several other lengths which play an important role in our study. These are the average separation length $l_{N P}$ between neighboring NPs, the nematic order parameter correlation length $\xi$, and the surface extrapolation length $d_{e}$. Their values are estimated by [2]

$$
l_{N P} \sim\left(\frac{4 \pi}{3 p}\right)^{1 / 3} r, \xi \sim \sqrt{K / f_{c}^{\prime \prime}}, d_{e} \sim \frac{K}{W_{0}} .
$$

Here (see Methods and Reference [2]), $K$ is the representative nematic Frank elastic constant, $f_{c}^{\prime \prime}=\frac{\partial^{2} f_{c}}{\partial S^{2}}$ is the second derivative of the nematic phase condensation free energy expressed at the equilibrium, and $w_{0}$ stands for the representative anchoring strength (see Methods). The correlation length estimates the length over which a locally-induced perturbation in the amplitude of nematic order persists. On the other hand, $d_{e}$ estimates the typical length scale on which the nematic director varies near a surface imposing an elastic distortion.

We next describe expected LC structural behaviour on decreasing temperature from the isotropic phase. We assume that the temperature is gradually decreased (i.e., mixtures are not "quenched" into the nematic phase). Firstly, we consider diluted samples, in which $l_{N P}>\xi$ in the whole temperature regime. For such conditions, the most probable configuration for $T>T_{I N}$ is schematically sketched in Figure 1. NPs act as seeds for paranematic (weakly ordered nematic) "islands" in the isotropic "sea", to which we henceforth refer to as clusters. The effective radius and volume of approximately spherical clusters equals to $r_{c l} \sim r+\xi$ and $v_{c l} \sim 4 \pi r_{c l}^{3} / 3$, respectively. Due to the weak anchoring, the average preferential paranematic orientation of each cluster exists, to which we henceforth refer to as the cluster director, that is aligned along some symmetry breaking direction. Namely, a paranematic ordering favours parallel alignment of LC molecules, which breaks the isotropic symmetry imposed by the homeotropic anchoring condition. On the other hand, the coupling between cluster directors is relatively weak. Consequently, the orientational distribution function of cluster directors is expected to be isotropic if $l_{N P} \gg \xi$. When the phase transition temperature is reached, the nematic order nucleated at NP-LC interfaces gradually pervades all the LC volume. This growth is slow enough (due to sluggish kinetics of domain growth) so that previously randomly aligned cluster directors realign along a common (in general domain) direction. In this concentration regime, the NP-imposed disorder strength is relatively weak. On increasing $p$, the "paranematic stiffness" increases and remains partially quenched in a weakly distorted metastable state on entering the nematic phase. We expect that the disorder strength increases with increasing $p$ in the regime $l_{N P}>2 r_{c l}$. At $l_{N P} \sim 2 r_{c l}$, the clusters are expected to enter "percolated" regime [23]. Note that at the percolation threshold [24] the structures typically exhibit fractal-like patterns and fractals in general fingertip "edge of chaos". In the regime $l_{N P}<2 r_{c l}$, the coupling between paranematic clusters becomes relatively strong (with respect to thermal fluctuations) even in the isotropic (i.e., paranematic phase). If this interaction is strong enough, it can partially realign neighbor clusters along a similar direction, and consequently, the disorder strength is decreased. 


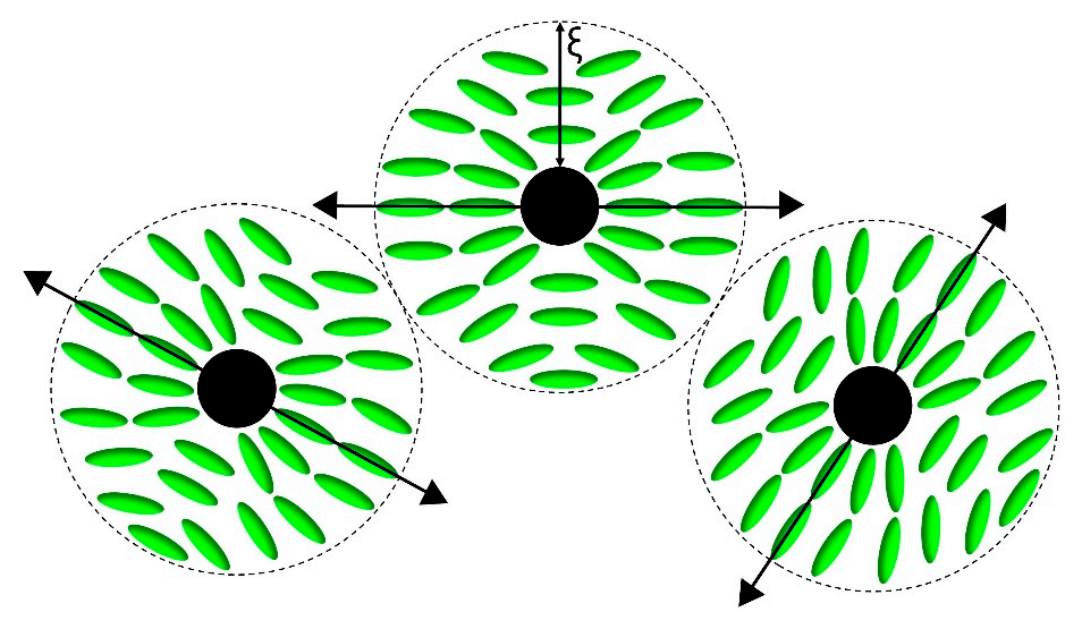

Figure 1. Schematic presentation of paranematic clusters embedded in the isotropic fluid. Double arrows indicate average orientation within each cluster.

Such a configuration could be treated as a binary system consisting of LC molecules and paranematic clusters. The corresponding effective free energy density could be approximately expressed as [25] $f^{(e f f)}=\left(1-p_{c l}\right) f_{L C}+p_{c l} f_{c l}+p_{c l}\left(1-p_{c l}\right) f_{\text {int }}$, where

$$
\begin{gathered}
f_{L C}=A_{0}\left(T-T^{*}\right) S^{2}-B S^{3}+C S^{4}, \\
f_{c l}=a_{0}\left(p_{c l}^{*}-p_{c l}\right) s_{c l}^{2}-b S_{c l}^{3}+c S_{c l}^{4}, \\
f_{\text {int }}=-W S_{c l} S .
\end{gathered}
$$

The terms $f_{L C}$ and $f_{c l}$ represent spatially averaged condensation contributions of the bulk-like LC component and clusters, respectively. Here, $S$ and $S_{c l}$ determine amplitudes of the respective nematic (paranematic) orderings; $A_{0}, B, C, a_{0}, b, c, T^{*}, p_{c l}^{*}$ are material dependent quantities; and

$$
p_{c l}=\frac{N v_{c l}}{V}=p \frac{v_{c l}}{v} \sim p\left(\frac{r_{c l}}{r}\right)^{3} .
$$

For example, for $\xi \sim r$ it follows $p_{c l} \sim 8 p$. The term $f_{c l}$ models the clusters as effectively lyotropic LC molecules diluted in an isotropic fluid. The interaction term $f_{\text {int }}$ models spatially averaged coupling between LC molecules and clusters, where the coupling constant $W$ is positive. This coupling favors mutually supporting the ordering of LC molecules and clusters. Therefore, in cases $S_{c l}>0$ the LC component experiences an external field-like coupling term $f_{\text {int }}=-w S$, where $w=W S_{c l}$ in "real" samples certainly exhibits a relatively strong spatial dependence. This introduces a certain degree of randomness in the system, in particular in the regime where percolation-like order of clusters is expected.

The fingerprint of this phenomenon could be non-monotonous $\Delta T(p)$ dependence observed in some LC mixtures, where examples are given in Table 1. In it, we list $\Delta T(p)$ measured in mixtures of different LCs and NPs $[17,18]$ in the diluted regime. The local minimum in $\Delta T(p)$ signals peaks in NP-imposed disorder strength. Namely, theoretical studies on the impact of random type disorder [26] on nematic ordering reveal that the bistability region decreases on increasing the disorder strength. A rough insight into the latter behaviour is also evident from a relatively simple phenomenological model presented in the Methods (see Equation (24)), where the dimensionless field $\sigma$ estimates NPs-induced disorder strength. On increasing $\sigma$, the nematic-paranematic coexistence temperature interval decreases in the regime $\sigma<0.5$ and vanishes at $\sigma=0.5$. 
Table 1. Measured critical behavior for mixtures of different liquid crystals and nanoparticles. In References $[17,18]$, they used $\mathrm{BaTiO}_{3}$ nanoparticles.

\begin{tabular}{cccccc}
\hline Sample & $\boldsymbol{T}_{\boldsymbol{I N}}$ & $\boldsymbol{T}^{*}$ & $\boldsymbol{T}^{* *}$ & $\boldsymbol{\Delta}$ & Reference \\
\hline 5OCB & 69.1 & 65.3 & & $\sim 3.8$ & {$[18]$} \\
$5 \mathrm{OCB}+0.1 \% \mathrm{NPs}$ & 68.4 & 68.1 & & $\sim 0.3$ & {$[18]$} \\
$5 \mathrm{OCB}+0.5 \% \mathrm{NPs}$ & 68.8 & 68.3 & & $\sim 0.5$ & {$[18]$} \\
$12 \mathrm{CB}$ & & 46.8 & 58.9 & 12.1 & {$[17]$} \\
$12 \mathrm{CB}+0.1 \% \mathrm{NPs}$ & & 53.4 & 58.9 & 5.5 & {$[17]$} \\
$12 \mathrm{CB}+0.2 \% \mathrm{NPs}$ & & 53.9 & 59.2 & 5.3 & {$[17]$} \\
$12 \mathrm{CB}+0.3 \% \mathrm{NPs}$ & & 54.4 & 58.6 & 4.2 & {$[17]$} \\
$12 \mathrm{CB}+0.4 \% \mathrm{NPs}$ & & 51.9 & 57.9 & 6 & {$[17]$} \\
\hline
\end{tabular}

\subsection{Impact of NPs on Average Nematic Ordering}

Polarized micro-Raman spectroscopy and optical birefringence measurements [19] revealed bulk-like equilibrium ordering in the nematic phase within the experimental resolution in the diluted regime $(p<0.01)$. However, the uniaxial order parameter displayed two different regimes on increasing $p$ as shown in Figure 2. In the first regime $\left(0<p \leq p_{c}\right)$, where $p_{c} \sim 0.001$, $S(p)$ monotonously decreased. However, in the second regime $\left(p_{c}<p<0.01\right)$, it gradually saturated to a roughly constant value.

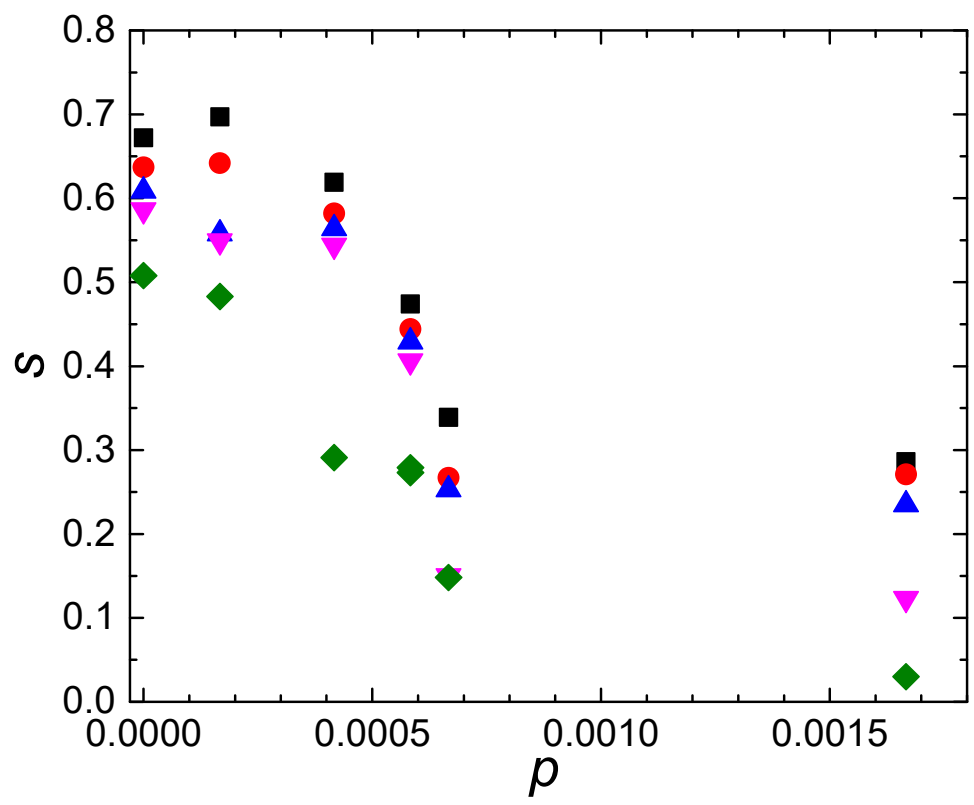

Figure 2. Nematic orientational order parameter $S$ as a function of nanoparticle concentration $p$ at different temperatures $\Delta T$ below the isotropic to nematic phase transition temperature $T_{I N}(\boldsymbol{\square}-\Delta T=19.4 \mathrm{~K} \pm$ $1.4 \mathrm{~K}, \bullet-\Delta T=15.0 \mathrm{~K} \pm 0.7 \mathrm{~K},-\Delta T=10.0 \mathrm{~K} \pm 0.7 \mathrm{~K}, \mathbf{\nabla}-\Delta T=5.0 \mathrm{~K} \pm 0.7 \mathrm{~K},-\Delta T=1.0 \mathrm{~K} \pm 0.7 \mathrm{~K})$.

To reproduce the basic mechanism behind this observation, we derive a simple mean-field-type expression at temperature $T=0$ for the nematic response in a randomly perturbed nematic phase. Our interest is in the structural behaviour which is expected to emerge in weakly disordered phases reached via a continuous symmetry breaking phase transition in which a spatially uniform orientational ordering is formed in the absence of disorder. We used the simplest possible modeling to reproduce the experimental observations reported in Reference [19]. In this study, homogeneously dispersed NPs enforce a relatively weak disorder. In the modeling, we assumed that each NP enforces in general a different local orientation which we roughly mimic by a kind of preferentially ordered random field of constant amplitude. Reasons for such randomness are described in the previous section. We used a 
Lebwohl-Lasher-type lattice modelling which despite its simplicity well describes general properties of nematic ordering [20-22].

We consider a $d$-dimensional lattice (we study cases $d=2$ and $d=3$ ) of $N_{n}$ nematic spins $\boldsymbol{n}_{i}$ pointing along a local direction of rod-like LC molecules at the site $r_{i}$ of a cubic lattice interacting by Lebwohl-Lasher interaction [20]. To take into account the nematic head-to-tail invariance, we introduce the traceless nematic tensor order parameter [2]

$$
\underline{\boldsymbol{q}}_{i}=\boldsymbol{n}_{i} \otimes \boldsymbol{n}_{i}-\underline{\mathbf{I}}^{(d)} / d,
$$

where $\underline{I}^{(d)}$ is the $d$-dimensional unit tensor and $\otimes$ marks the tensor product. We define the global nematic order parameter

$$
\underline{\boldsymbol{Q}}=s^{(d)}\left(\boldsymbol{n}_{g} \otimes \boldsymbol{n}_{g}-\underline{\boldsymbol{I}}^{(d)} / d\right)=\left\langle\underline{\boldsymbol{q}}_{i}\right\rangle,
$$

of the system, where $\langle\ldots\rangle$ stands for the spatial average. The global nematic director $n_{g}$ points along the average nematic direction in the system and $s^{(d)}$ stands for the global uniaxial nematic order parameter. Furthermore, we set that some randomly chosen sites are occupied by NPs, where their volume concentration is determined by $p \in[0,1]$. In our modeling, we consider dilute regimes characterized by $p<0.01$. We set that a NP located at the $i$ th site enforces a local "easy direction" determined by the tensor

$$
\underline{\boldsymbol{q}}_{i}^{(N P)}=\boldsymbol{e}_{i} \otimes \boldsymbol{e}_{i}-\underline{\mathbf{I}}^{(d)} / d,
$$

and orientations of unit vectors $\boldsymbol{e}_{i}$ are distributed according to some probability distribution $P\left(\boldsymbol{e}_{i}\right)$.

Taking into account Equations (5)-(6), we obtain

$$
s^{(d)}=\frac{1}{d-1}\left\langle d\left(\boldsymbol{n}_{i} \cdot \boldsymbol{n}_{g}\right)^{2}-1\right\rangle .
$$

Therefore, for $d=2$ and $d=3$ it follows

$$
\begin{gathered}
s^{(2)}=\left\langle 2\left(\boldsymbol{n}_{i} \cdot \boldsymbol{n}_{g}\right)^{2}-1\right\rangle=\left\langle\cos \left(2 \theta_{i}\right)\right\rangle, \\
s^{(3)}=\left\langle\frac{1}{2}\left(3\left(\boldsymbol{n}_{i} \cdot \boldsymbol{n}_{g}\right)^{2}-1\right)\right\rangle=\left\langle\frac{1}{2}\left(3 \cos ^{2} \theta_{i}-1\right)\right\rangle .
\end{gathered}
$$

For later convenience, we introduce also

$$
\begin{gathered}
s_{N P}^{(2)}=2\left(\boldsymbol{e}_{i} \cdot \boldsymbol{n}_{g}\right)^{2}-1=\cos \left(2 \vartheta_{i}\right), \\
s_{N P}^{(3)}=\frac{1}{2}\left(3\left(\boldsymbol{e}_{i} \cdot \boldsymbol{n}_{g}\right)^{2}-1\right)=\frac{1}{2}\left(3 \cos ^{2} \vartheta_{i}-1\right) .
\end{gathered}
$$

The angles $\theta_{i}$ and $\vartheta_{i}$ are defined as

$$
\begin{gathered}
\theta_{i}=\operatorname{ArcCos}\left(\boldsymbol{n}_{i} \cdot \boldsymbol{n}_{g}\right), \\
\vartheta_{i}=\operatorname{ArcCos}\left(\boldsymbol{e}_{i} \cdot \boldsymbol{n}_{g}\right) .
\end{gathered}
$$

Note that $\left\{s^{(2)}, s_{N P}^{(2)}\right\} \in[-1,1]$ and $\left\{s^{(3)}, s_{N P}^{(3)}\right\} \in[-1 / 2,1]$. Furthermore, $s^{(d)}=1\left(s_{N P}^{(d)}=1\right)$ fingerprints that the nematic director field (NP enforced easy direction) is strictly aligned along $\boldsymbol{n}_{g}$. Isotropic distribution of $\boldsymbol{n}_{i}$ yields $s^{(d)}=0$. Similarly, for isotropic distribution of $\boldsymbol{e}_{i}$, it follows $\left\langle s_{N P}^{(d)}\right\rangle=0$.

In the spirit of classical mean-field approaches [21,27], we replace the molecular field $\underline{\boldsymbol{B}}_{i}$ (see Equation (17)) which is introduced in the Methods by an effective molecular field acting on each nematic spin:

$$
\underline{\boldsymbol{B}}=J \underline{Q}+w \underline{\boldsymbol{q}}_{i}^{(N P)} .
$$


Here, $J=N_{n n} J_{0}, J_{0}$ describes the interaction between nearby nematic spins, $N_{n n}$ stands for the number of first neighbors, $w=p w_{0}$, and $w_{0}$ represents the local "impurity" field strength (see Methods).

Next, we assume that a local nematic orientation is determined by the effective molecular field [21,27]. Hence, we request that the frames of $\underline{\boldsymbol{B}}$ and $\underline{\boldsymbol{q}}_{i}$ coincide (are parallel) [21], i.e., $\underline{\boldsymbol{q}}_{i}=A \underline{\boldsymbol{B}}$. We determine the constant $A$ from the condition $\operatorname{Tr}\left(\underline{\boldsymbol{q}}_{i} \underline{\boldsymbol{q}}_{i}\right)=C^{(d)}$, where $C^{(2)}=1 / 2$ and $C^{(3)}=2 / 3$ for $d=2$ and $d=3$, respectively. Therefore, $\underline{\boldsymbol{q}}_{i}=\left(J \underline{\boldsymbol{Q}}+w \underline{\boldsymbol{q}}_{i}^{(N P)}\right) / \sqrt{\left(J \mathcal{s}^{(d)}\right)^{2}+w^{2}+2 J w \boldsymbol{s}^{(d)}}$, and it follows

$$
\boldsymbol{n}_{g} \cdot \underline{\underline{q}}_{i} \boldsymbol{n}_{g}=\frac{J s^{(d)}+w s_{N P}^{(d)}}{\sqrt{\left(J s^{(d)}\right)^{2}+w^{2}+2 J w s s_{N P}^{(d)}}} .
$$

Finally, we take the spatial average of Equation (12), which yields the self-consistent integral equation for $s^{(d)}$ for a given probability distribution $P\left(\boldsymbol{e}_{i}\right)$ :

$$
s^{(d)}=\frac{1}{\Omega^{(d)}} \int P\left(\boldsymbol{e}_{i}\right) \frac{J s^{(d)}+w s_{N P}^{(d)}}{\sqrt{\left(J \mathcal{S}^{(d)}\right)^{2}+w^{2}+2 J w s_{N P}^{(d)}}} d \Omega^{(d)} .
$$

The integration is carried over all possible orientations $\boldsymbol{e}_{i}, \Omega^{(d)}=\int d \Omega^{(d)}$, and $\frac{1}{\Omega^{(d)}} \int P d \Omega^{(d)}=1$. In our approximate treatment, we assume a cylindrically symmetric distribution of $\boldsymbol{e}_{i}$ values about $\boldsymbol{n}_{g}$. Consequently,

$$
\begin{gathered}
s^{(2)}=\frac{1}{\pi} \int_{0}^{\pi} P\left(\cos \vartheta_{i}\right) \frac{J s^{(2)}+w s_{N P}^{(2)}}{\sqrt{\left(J s^{(2)}\right)^{2}+w^{2}+2 J w s_{N P}^{(2)}}} d \vartheta_{i}, \\
s^{(3)}=\frac{1}{2} \int_{-1}^{1} P\left(\cos \vartheta_{i}\right) \frac{J s^{(3)}+w s_{N P}^{(3)}}{\sqrt{\left(J s^{(3)}\right)^{2}+w^{2}+2 J w s_{N P}^{(3)}}} d \cos \vartheta_{i} .
\end{gathered}
$$

Note, that Equation (13) yield $s^{(d)}=1$ for $w=0$. Furthermore, in the limit $w \rightarrow \infty$ and the isotropic distribution $P$ it follows $s^{(d)}=0$.

In Figure 3, we plot $S=s^{(3)}$ as a function of $w=p w_{0}$ for different distributions $P$ and $d=3$. In the calculations, we impose a step-like distribution $P\left(\vartheta \leq \vartheta^{(\max )}\right)=P_{0}$ and $P\left(\vartheta>\vartheta^{(\max )}\right)=0$, where $P_{0}$ is a constant. The $S(p)$ dependence monotonously decreases on increasing $p$, displaying a relatively steep crossover between the two regimes, characterized by significantly different characteristic values of S. Saturated value of $S_{S}$ in the second (plateau) regime monotonously decreases with $\vartheta^{(\max )}$ and is finite for $\vartheta^{(\max )}<\pi / 2$. Note that the behaviour shown in Figure 3 is relatively robust with respect to $P$ shape. For example, in Figure 3c, we compare $S(p)$ behaviors for a step-like and Gaussian distribution. One sees that $S_{s}$ well fingerprints the essential property of $P$.

We stress that in our simulations $w=w_{0} p$ is increased either by increasing $w_{0}$ or $p$. However, our preliminary semi-microscopic lattice simulations using interactions at finite temperatures reveal that experimentally observed crossover behavior on varying $p$ could be reproduced if the anchoring strength $w_{0}$ is relatively weak. To estimate the anchoring strength in experiments, we use a simple mesoscopic model where the corresponding free energy contributions are given by Equation (20) (see Methods). Experimental measurements reveal negligible shifts in $\Delta T_{I N}(p)=T_{I N}-T_{I N}(p)$ on varying $p$. Our mesoscopic estimate (see Equation (26a)) reveals that elastic and NP-LC interface contributions tend to decrease and increase $\Delta T_{I N}(p)$, respectively. We set that the contradicting effects cancel each other, i.e., $\Delta T_{I N}(p)=0$. It follows

$$
p \frac{\xi_{d}^{2}}{d_{I N} r} \sim 1
$$


where $d_{I N}=d_{e}\left(T=T_{I N}\right), \xi_{d}$ estimates a typical linear length over which distortions in the nematic director field are realized, and we assume $\bar{P}_{2} \sim 1$ (see Methods, Equation (25)). By setting $\xi_{d} \sim d_{I N}$, we obtain (see Equation (2)) $w_{0} \sim \frac{p K}{r}$. For $p \sim 0.01, K \sim 10^{-11} \mathrm{~J} / \mathrm{m}$, and $r \sim 25 \mathrm{~nm}$ [19] it follows $w_{0} \sim 10^{-5} \mathrm{~J} / \mathrm{m}^{2}$, confirming a relatively weak anchoring regime. Note that the interaction between NPs and LCs depends on material properties of both components and also on NP surface treatment [28].
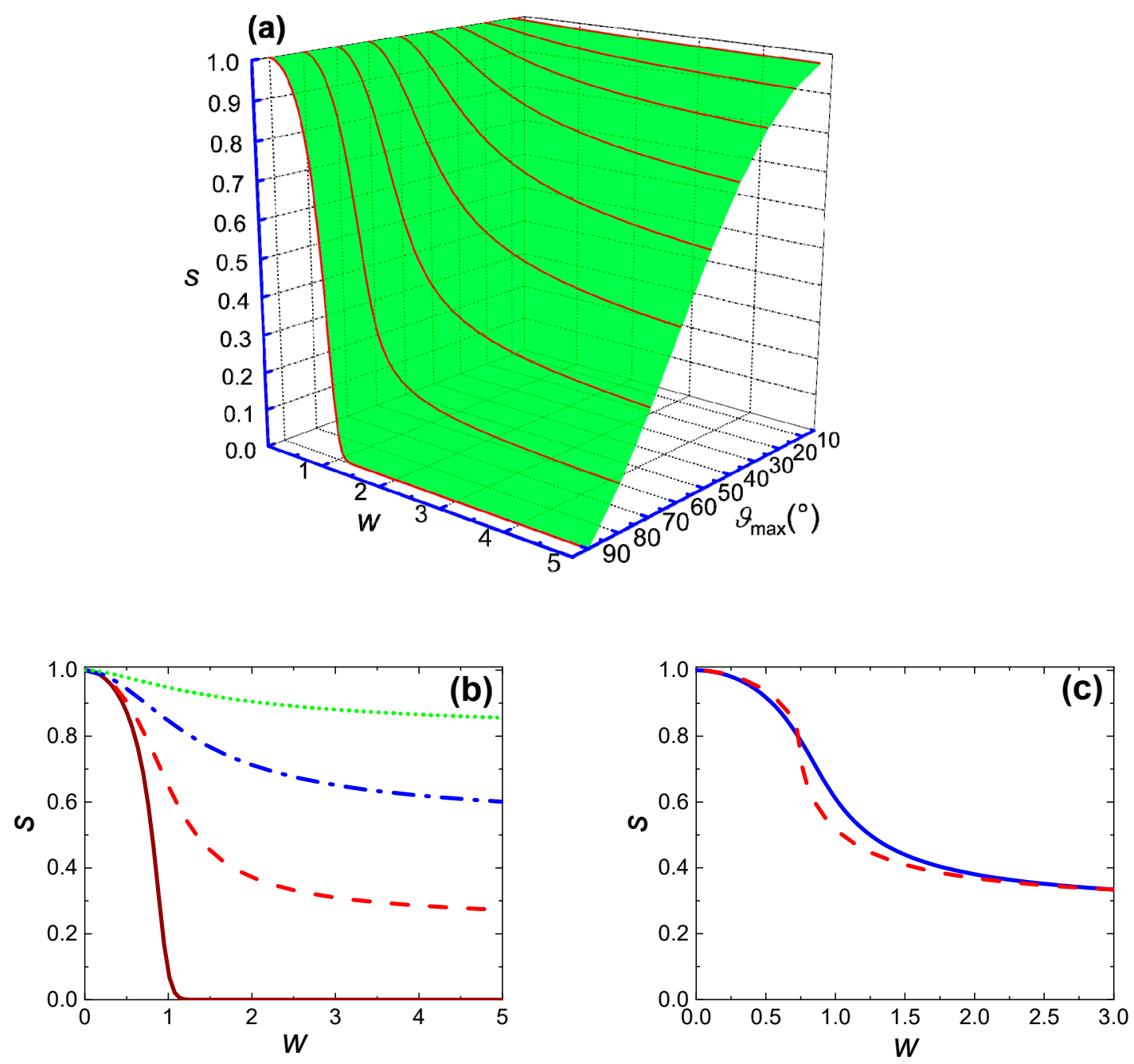

Figure 3. (a) Order parameter $S$ variation as a function of $w$ and $\vartheta_{\max }$. (b) $S(w)$ variation for several values of $\vartheta_{\max }$; full line: $\vartheta_{\max }=\pi / 2$, dashed line: $\vartheta_{\max }=7 \pi / 18$, dash-dotted line: $\vartheta_{\max }$ $=\pi / 3$, dotted line: $\vartheta_{\max }=2 \pi / 9$. (c) Comparison of $S(w)$ dependences for a step-like (full line) and the Gaussian probability distribution (dashed line). The step-like distribution is described by $P\left(\vartheta \leq \vartheta^{(\text {max })}\right)=P_{0}$ and $P\left(\vartheta>\vartheta^{(\text {max })}\right)=0$, where $P_{0}$ is a constant, and the Gaussian distribution is described by $P=P_{G}(\vartheta)=C e^{-\vartheta^{2} / 2 \sigma^{2}}$, where $C$ is the normalization constant, and $\sigma$ is the standard deviation. Both probability distributions must be normalized on the interval from $\vartheta=0$ to $\vartheta=\pi$. We chose the free parameters for both distributions in such a way to roughly match the observed drop in experimentally measured $S(p)$ dependence. For the step-like distribution, we obtain $\vartheta_{\max }=0.387 \pi$, while for the Gaussian distribution, $\sigma=0.798$ and $C=0.25$.

\section{Conclusions}

Of interest were dilute mixtures of nematic liquid crystals and nanoparticles, where NPs enforce a relatively weak disorder. 
Our research was motivated by recent experimental observations [17-19] in such dilute mixtures where nematic structural properties were studied on varying the concentration $p$ of NPs. It was observed $[17,18]$ that the temperature width of the isotropic-nematic coexistence region exhibits a non-monotonous behaviour. Furthermore, the global nematic order parameter of mixtures exhibits the crossover [19] between two quantitatively different bulk-like nematic ordering regimes. We claim that these features emerge due to NP-induced disorder. Namely, owing to the continuous symmetry breaking origin of I-N phase transition, the nematic order is strongly susceptible to even relatively weak perturbations.

We used minimal models to discuss these phenomena. We considered a dilute NP concentration regime where phase separation is not expected. We assumed that NPs are relatively weakly coupled with the surrounding LC order. Consequently, in the isotropic (or weakly paranematic) phase, NPs nucleate paranematic clusters, and each of them in general displays a preferential ordering direction. One expects three different regimes depending on the relative value of $l_{N P}$ (the mean separation of NPs) and $r_{c l}$ (the effective radius of clusters). For $l_{N P}>2 r_{c l}$, the clusters are essentially decoupled. In the regime $l_{N P} \sim 2 r_{c l}$, the clusters are expected to form a percolation-type structure, which in general display fractal properties and are consequently relatively disordered. For $l_{N P}<2 r_{c l}$, the clusters relatively strongly interact, which suppresses the thermal fluctuation of clusters. Consequently, there is a strong tendency to align clusters along a common direction. As a result, the degree of disorder, which is experienced by LCs, displays the non-monotonous behaviour on varying $p$. Note that a similar $p$-driven disorder strength impact is observed in $T_{I N}(p)$ non-monotonous dependence in aerosil-LC mixtures. Note that in these mixtures the interaction between NPs and LC molecules is relatively strong due to the formation of covalent bonds. On increasing $p$, the I-N phase transition temperature first decreases. In the second regime, it increases and again decreases above $p=p_{\max }$, where the local minimum is observed in the $T_{I N}(p)$ dependence. The maximum at $p=p_{\max }$ is attributed to the peak in disorder strength. For values of $p>p_{\max }$, the disorder strength again decreases due to elastic strain-driven rearrangement of the aerosil network. Namely, the network rearrangement enables partial relaxation of the LC elastic strain.

In more detail, we analyzed weak disorder-driven crossover behaviour of the nematic order parameter $s$ on varying $p$ deep in the nematic phase. We used a Lebwohl-Lasher-type lattice semi-microscopic model. We set that NPs locally enforce a preferential orientational order, where the distribution of the corresponding easy directions is determined by the probability distribution $P$. We used a mean field-type approach and assumed that nematic spins locally align along the effective local field and derived the corresponding self-consistent equation for S. A simple cylindrically symmetric step-like shape of $P$ yields the $p$-driven crossover behaviour which is observed experimentally. This shape in $P$ roughly mimics cases where NPs generated preferred local orientations which are on average aligned along the global nematic order. Our analysis reveals that $S(p)$ behaviour is relatively robust with respect to details describing $P$. Note that in the experiments $P$ distribution is expected to quantitatively change on varying $p$. The modeling of this rearrangement mechanism is the topic of our next research.

\section{Methods}

\subsection{Semi-Microscopic Modeling}

We use a Lebwohl-Lasher-type lattice model [20] to describe nematic structures perturbed by impurities, which in our description mimic randomly dispersed nanoparticles. We consider a lattice consisting of nematic spins $\boldsymbol{n}_{i}$. In our approximate treatment, we set that impurities effectively act as an external field [21,29], which is in general spatially-dependent. We express the total energy of the system $[20-22,29]$ as

$$
W=-\frac{1}{2} \sum_{i=1}^{N_{n}} \sum_{j=1}^{N_{n}} J_{i j} \operatorname{Tr}\left(\underline{\boldsymbol{q}}_{i} \underline{\boldsymbol{q}}_{j}\right)-\sum_{i=1}^{N_{n}} w_{i} \operatorname{Tr}\left(\underline{\boldsymbol{q}}_{i} \underline{\boldsymbol{q}}_{i}^{(N P)}\right) .
$$


Here, $\operatorname{Tr}$ stands for the trace operator, and the sums run over the lattice sites. The first term describes the interaction among nematic spins. We set $J_{i j}=J_{0}>0$ among the first neighbors, $J_{i j}=0$ for the other pairs and $J_{i i}=0$. This choice models short-range interaction, enforcing parallel alignment of nematic spins. The second term, which was originally introduced by Sluckin et al. [29] to study random-anisotropy-nematic (RAN) interactions, mimics the interaction between nematic spins and impurities. The quantity $w_{i}$ stands for the effective NP-generated local interaction strength. We set $w_{i}=w_{0}>0$ at the sites where NPs are present and $w_{i}=0$ otherwise. Hence, in the limit $w \rightarrow \infty$ the RAN interaction locally enforces $\boldsymbol{e}_{i}=\boldsymbol{n}_{i}$.

One can express Equation (15) as

$$
W=-\sum_{i=1}^{N_{n}} \operatorname{Tr}\left(\underline{\boldsymbol{B}}_{i} \underline{\boldsymbol{q}}_{i}\right)
$$

where we introduce the molecular field tensor [21] as

$$
\underline{\boldsymbol{B}}_{i}=\sum_{j=1}^{N_{n}} J_{i j} \underline{\boldsymbol{q}}_{j}+w_{i} \underline{\boldsymbol{q}}_{i}^{(N P)} .
$$

\subsection{Mesoscopic Modeling}

We consider the impact of the NP-LC interface interaction on paranematic and nematic order in thermotropic LC. At the mesoscopic level, we describe nematic orientational order for $d=3$ by the tensor nematic order parameter [2]

$$
\underline{Q}=S\left(\boldsymbol{n} \otimes \boldsymbol{n}-\underline{\mathbf{I}}^{(3)} / 3\right),
$$

where $s \in[-1 / 2,1]$ is the uniaxial order parameter, and $n$ is the nematic director field pointing along the local uniaxial order.

We express the free energy of the LC-NP mixture in terms of the nematic tensor order parameter in the diluted regime as [2]

$$
F=\int\left(f_{c}+f_{e}\right) d^{3} r+\int f_{i} d^{2} r
$$

The first integral runs over the LC material and the second over LC-NP interfaces. The condensation $\left(f_{c}\right)$, elastic $\left(f_{e}\right)$, and interface $\left(f_{i}\right)$ free energy density contributions are expressed as

$$
\begin{gathered}
f_{c}=\frac{3 A_{0}}{2}\left(T-T^{*}\right) \operatorname{Tr} \underline{Q}^{2}-9 B \operatorname{Tr} \underline{Q}^{3}+\frac{9 C}{4}\left(\operatorname{Tr} \underline{Q}^{2}\right)^{2}, \\
f_{e}=L \operatorname{Tr}(\nabla \underline{Q})^{2}, \\
f_{e}=-w_{0} v \cdot \underline{Q} v .
\end{gathered}
$$

The quantities $A_{0}, B$, and $C$ are positive material constants, and $T^{*}$ is the isotropic phase supercooling temperature. In bulk LC, the isotropic-nematic phase transition takes place at $T_{I N}=$ $T^{*}+\frac{B^{2}}{4 A_{0} \mathrm{C}}$. The condensation term determines the equilibrium degree of nematic ordering below $T_{I N}$, which equals to

$$
S_{e q}=s_{0} \frac{3+\sqrt{9-8 \tau}}{4}
$$

Here

$$
\tau=\frac{T-T^{*}}{T_{I N}-T^{*}}, \quad s_{0}=S_{e q}\left[T=T_{I N}\right]=\frac{B}{2 C^{\prime}},
$$

where $\tau$ stands for the dimensionless temperature. 
The elastic term is expressed in the single elastic constant approximation. Here, $L>0$ is the representative bare (i.e., independent of $T$ ) nematic elastic constant, and the corresponding temperature-dependent nematic Frank representative elastic constant is given by $K \sim L S^{2}$. This term enforces a spatially uniform order.

The interface term determines the condition at the LC-NP interface. It is weighted by the positive constant $w_{0}$, and $v$ is the normal surface of an infinitesimal interface area. This modeling favours homeotropic anchoring, i.e, local alignment of $n$ along $v$.

For latter convenience, we express the nematic correlation length $\xi$ and the interface extrapolation length at $T=T_{I N}$

$$
\xi_{I N}=\xi\left[T=T_{I N}\right] \equiv \sqrt{\frac{L}{A_{0}\left(T_{I N}-T^{*}\right)}}, \quad d_{I N}=d_{e}\left[T=T_{I N}\right]=\frac{L s_{0}}{w_{0}} .
$$

Next, we estimate the effective free energy of a mixture. For this purpose, we introduce the scaled nematic order parameter $q=S / s_{0}$, and we assume that nematic order amplitude is uniaxial and spatially homogeneous. Furthermore, we assume that typical NP-enforced distortions in $n$ are resolved on the length scale $\xi_{d}$. Consequently, $\left\langle f_{e}\right\rangle \sim \frac{L S^{2}}{\tilde{\xi}_{d}^{2}}$, where $\langle\ldots\rangle$ denotes the spatial average. It follows

$$
\frac{f}{f_{0}} \sim \tau^{(e f f)} q^{2}-2 q^{3}+q^{4}-\sigma q
$$

where

$$
\tau^{(e f f)}=\tau+\xi_{I N}^{2} / \xi_{d}^{2}, \quad \sigma=p \overline{P_{2}} \xi_{I N}^{2} /\left(d_{I N} r\right)
$$

stand for the effective dimensionless temperature and effective dimensionless NP-LC interaction, and $f_{0}=A_{0}\left(T_{I N}-T^{*}\right) s_{0}^{2}$. In expressing the latter term, we set $\int f_{i} d^{2} \boldsymbol{r} \sim-N a w_{0} \overline{P_{2}}, a=4 \pi \mathrm{r}^{2}$ is a NP's surface area, $\overline{P_{2}}=\left\langle\left(3(n . v)^{2}-1\right) / 2\right\rangle_{N P}$, and $\langle\ldots\rangle_{N P}$ stands for an average over an average NP's surface area. The phase behavior emerging from Equation (24) is as follows. For $\sigma \in[0,0.5]$, the isotropic-nematic $(\sigma=0)$ and paranematic-nematic $(\sigma>0)$ are determined by the condition $\tau^{(e f f)}=\tau_{I N}^{(e f f)} \equiv 1+\sigma$. The corresponding dimensional critical temperature $\tau_{I N}$ and amplitude $q_{I N}$ of the nematic order parameter are expressed as

$$
\begin{gathered}
\Delta \tau_{I N}=\tau_{I N}(p)-\tau_{I N}(0)=\frac{p \overline{P_{2}} \xi_{I N}^{2}}{d_{I N} r}-\frac{\xi_{I N}^{2}}{\xi_{d}^{2}}, \\
q_{I N}=\frac{1 \pm \sqrt{1-2 \sigma}}{2} .
\end{gathered}
$$

Here $+(-)$ determines the state just above (below) the critical temperature. For $\sigma>0.5$, the paranematic-nematic transition on varying temperature is gradual.

Author Contributions: D.Č. carried our simulations. S.K. has written the article. All authors have been involved in developing the model to reproduce experimental observations.

Funding: This research was funded by Slovenian Research Agency (ARRS), grant numbers P1-0099, PR-08970, P1-0125 and the Hellenic Foundation for Research and Innovation (HFRI) and the General Secretariat for Research and Technology (GSRT), under the HFRI PhD Fellowship grant (No. 1318).

Conflicts of Interest: The authors declare no conflict of interest.

\section{References}

1. Balazs, A.C.; Emrick, T.; Russell, T.P. Nanoparticle Polymer Composites: Where Two Small Worlds Meet. Science 2006, 314, 1107-1110. [CrossRef] [PubMed] 
2. Lavrentovich, O.D.; Kleman, M. Soft Matter Physics: An Introduction; Kleman, M., Lavrentovich, O.D., Eds.; Partially Ordered Systems; Springer: New York, NY, USA, 2004; ISBN 978-0-387-95267-3.

3. Palffy-Muhoray, P. The diverse world of liquid crystals. Phys. Today 2007, 60, 54-60. [CrossRef]

4. Iglič, A.; Kralj-Iglič, V.; Drobne, D. Nanostructures in Biological Systems: Theory and Applications; Pan Stanford: Singapore, 2015; ISBN 9789814267205.

5. Crawford, G.P.; Žumer, S. Liquid Crystals In Complex Geometries: Formed by Polymer and Porous Networks; Crawford, G.P., Žumer, S., Eds.; Taylor and Francis: New York, NY, USA, 1996; ISBN 0-7484-0464-3.

6. Iannacchione, G.S.; Garland, C.W.; Mang, J.T.; Rieker, T.P. Calorimetric and small angle x-ray scattering study of phase transitions in octylcyanobiphenyl-aerosil dispersions. Phys. Rev. E 1998, 58, 5966-5981. [CrossRef]

7. Kurik, M.V.; Lavrentovich, O.D. Defects in liquid crystals: Homotopy theory and experimental studies. Sov. Phys. Uspekhi 1988, 31, 196-224. [CrossRef]

8. Bellini, T.; Radzhihovsky, L.; Toner, J.; Clark, N.A. Universality and Scaling in the Disordering of a Smectic Liquid Crystal. Science 2001, 294, 1074-1079. [CrossRef] [PubMed]

9. Bellini, T.; Buscaglia, M.; Chiccoli, C.; Mantegazza, F.; Pasini, P.; Zannoni, C. Nematics with Quenched Disorder: How Long Will It Take to Heal? Phys. Rev. Lett. 2002, 88, 245506. [CrossRef] [PubMed]

10. León, N.; Korb, J.-P.; Bonalde, I.; Levitz, P. Universal Nuclear Spin Relaxation and Long-Range Order in Nematics Strongly Confined in Mass Fractal Silica Gels. Phys. Rev. Lett. 2004, 92, 195504. [CrossRef] [PubMed]

11. Park, S.; Leheny, R.L.; Birgeneau, R.J.; Gallani, J.-L.; Garland, C.W.; Iannacchione, G.S. Hydrogen-bonded silica gels dispersed in a smectic liquid crystal: A random field XY system. Phys. Rev. E 2002, 65, 050703. [CrossRef] [PubMed]

12. Imry, Y.; Ma, S. Random-Field Instability of the Ordered State of Continuous Symmetry. Phys. Rev. Lett. 1975, 35, 1399-1401. [CrossRef]

13. Chakrabarti, J. Simulation Evidence of Critical Behavior of Isotropic-Nematic Phase Transition in a Porous Medium. Phys. Rev. Lett. 1998, 81, 385-388. [CrossRef]

14. Ranjkesh, A.; Ambrožič, M.; Kralj, S.; Sluckin, T.J. Computational studies of history dependence in nematic liquid crystals in random environments. Phys. Rev. E 2014, 89, 022504. [CrossRef]

15. Larkin, A.I. Effect of inhomogeneities on the structure of the mixed state of superconductors. Sov. Phys. JETP 1970, 31, 784.

16. Feldman, D.E. Quasi-long range order in glass states of impure liquid crystals, magnets, and superconductors. Int. J. Mod. Phys. B 2001, 15, 2945-2976. [CrossRef]

17. Rzoska, S.J.; Starzonek, S.; Drozd-Rzoska, A.; Czupryński, K.; Chmiel, K.; Gaura, G.; Michulec, A.; Szczypek, B.; Walas, W. Impact of $\mathrm{BaTiO}_{3}$ nanoparticles on pretransitional effects in liquid crystalline dodecylcyanobiphenyl. Phys. Rev. E 2016, 93, 020701. [CrossRef] [PubMed]

18. Starzonek, S.; Rzoska, S.J.; Drozd-Rzoska, A.; Czupryński, K.; Kralj, S. Impact of ferroelectric and superparaelectric nanoparticles on phase transitions and dynamics in nematic liquid crystals. Phys. Rev. E 2017, 96, 022705. [CrossRef] [PubMed]

19. Kyrou, C.; Kralj, S.; Panagopoulou, M.; Raptis, Y.; Nounesis, G.; Lelidis, I. Impact of spherical nanoparticles on nematic order parameters. Phys. Rev. E 2018, 97, 042701. [CrossRef]

20. Lebwohl, P.A.; Lasher, G. Nematic-Liquid-Crystal Order-A Monte Carlo Calculation. Phys. Rev. A 1972, 6, 426-429. [CrossRef]

21. Buček, S.; Kralj, S.; Sluckin, T.J. Hysteresis in Two-Dimensional Liquid Crystal Models. Adv. Condens. Matter Phys. 2015, 2015, 1-10. [CrossRef]

22. Luckhurst, G.R.; Sluckin, T.J.; Zewdie, H.B. Computer simulation studies of anisotropic systems. Mol. Phys. 1986, 59, 657-678. [CrossRef]

23. Ambrožič, M.; Kralj, S. Field percolation-switching in soft ternary anisotropic system. Phys. A Stat. Mech. its Appl. 2019, 520, 11-25. [CrossRef]

24. Stauffer, D.; Aharony, A. Introduction to Percolation Theory; Taylor \& Francis: Abingdon, UK, 1985, ISBN 978-0-203-29042-2.

25. Popa-Nita, V.; van der Schoot, P.; Kralj, S. Influence of a random field on particle fractionation and solidification in liquid-crystal colloid mixtures. Eur. Phys. J. E 2006, 21, 189-197. [CrossRef] [PubMed]

26. Popa-Nita, V. Statics and kinetics at the nematic-isotropic interface in porous media. Eur. Phys. J. B 1999, 12, 83-90. [CrossRef] 
27. Lopatina, L.M.; Selinger, J.V. Maier-Saupe-type theory of ferroelectric nanoparticles in nematic liquid crystals. Phys. Rev. E 2011, 84, 041703. [CrossRef] [PubMed]

28. Cordoyiannis, G.; Kurihara, L.K.; Martinez-Miranda, L.J.; Glorieux, C.; Thoen, J. Effects of magnetic nanoparticles with different surface coating on the phase transitions of octylcyanobiphenyl liquid crystal. Phys. Rev. E 2009, 79, 011702. [CrossRef] [PubMed]

29. Cleaver, D.J.; Kralj, S.; Sluckin, T.J.; Allen, M.P. The random anisotropy nematic spin model. In Liquid Crystals in Complex Geometries: Formed by Polymer and Porous Networks; Crawford, G.P., Žumer, S., Eds.; Taylor and Francis: New York, NY, USA, 1996; pp. 467-481, ISBN 0-7484-0464-3.

(C) 2019 by the authors. Licensee MDPI, Basel, Switzerland. This article is an open access article distributed under the terms and conditions of the Creative Commons Attribution (CC BY) license (http://creativecommons.org/licenses/by/4.0/). 